\title{
MGAT2 deficiency ameliorates high-fat diet-induced obesity and insulin resistance by inhibiting intestinal fat absorption in mice
}

Takuma Tsuchida ${ }^{*}$, Sayaka Fukuda ${ }^{1}$, Hisanori Aoyama ${ }^{2}$, Nobuhiko Taniuchi ${ }^{1}$, Tomomi Ishihara ${ }^{1}$, Noriko Ohashi ${ }^{1}$, Hiroko Sato ${ }^{3}$, Koji Wakimoto ${ }^{4}$, Masaharu Shiotani ${ }^{1}$ and Akira Oku ${ }^{1}$

\begin{abstract}
Background: Resynthesis of triglycerides in enterocytes of the small intestine plays a critical role in the absorption of dietary fat. Acyl-CoA:monoacylglycerol acyltransferase-2 (MGAT2) is highly expressed in the small intestine and catalyzes the synthesis of diacylglycerol from monoacylglycerol and acyl-CoA. To determine the physiological importance of MGAT2 in metabolic disorders and lipid metabolism in the small intestine, we constructed and analyzed Mgat2-deficient mice.

Results: In oral fat tolerance test (OFT), Mgat2-deficient mice absorbed less fat into the circulation. When maintained on a high-fat diet (HFD), Mgat2-deficient mice were protected from HFD-induced obesity and insulin resistance. Heterozygote $\left(\mathrm{Mgat}^{+/-}\right)$mice had an intermediate phenotype between $\mathrm{Mgat}^{+/+}$and $\mathrm{Mgat2}^{-/-}$and were partially protected from metabolic disorders. Despite of a decrease in fat absorption in the Mgat2-deficient mice, lipid levels in the feces and small intestine were comparable among the genotypes. Oxygen consumption was increased in the Mgat2-deficient mice when maintained on an HFD. A prominent upregulation of the genes involved in fatty acid oxidation was observed in the duodenum but not in the liver of the Mgat2-deficient mice.
\end{abstract}

Conclusion: These results suggest that MGAT2 has a pivotal role in lipid metabolism in the small intestine, and the inhibition of MGAT2 activity may be a promising strategy for the treatment of obesity-related metabolic disorders.

Keywords: Acyl-coenzyme A:monoacylglycerol acyltransferase (MGAT), Obesity, Insulin resistance, Triglyceride, Enterocyte, Fatty acid oxidation

\section{Background}

Intestinal fat absorption involves hydrolysis of dietary triglycerides to 2-monoacylglycerol and fatty acids in the lumen by pancreatic lipase [1]. These hydrolysis products are taken up by enterocytes, and triglycerides are resynthesized through the monoacylglycerol pathway, which is catalyzed by acyl-CoA:monoacylglycerol acyltransferase (MGAT) and acyl-CoA:diacylglycerol acyltransferase (DGAT). Another pathway involved in triglyceride synthesis is the glycerol 3-phosphate pathway, a de novo pathway that is present in most tissues [2]. In the small intestinal mucosa, the monoacylglycerol pathway

\footnotetext{
* Correspondence: tsuchida.takuma@mx.mt-pharma.co.jp

'Department I, Pharmacology Research Laboratories II, Research Division, Mitsubishi Tanabe Pharma Corporation, 2-2-50, Kawagishi, Toda-shi, Saitama 335-8505, Japan

Full list of author information is available at the end of the article
}

accounts for $70-80 \%$ of triglyceride resynthesis after a meal because of the large amount of 2-monoacylglycerol released from dietary fat [3-5]. The newly formed triglycerides are then incorporated into chylomicrons with other lipids for secretion into the blood and transport to other tissues such as the liver and adipose tissue.

MGAT acylates monoacylglycerol to yield diacylglycerol. Three isoforms of MGAT enzymes, MGAT1, MGAT2, and MGAT3, have been identified thus far [6-9]. MGAT1 is mainly expressed in the stomach and kidney and expressed at lower levels in adipose tissue and the liver, but is absent in the small intestine [9]. MGAT2 and MGAT3 are highly expressed in the small intestine $[6-8,10]$. MGAT2 is expressed in both humans and rodents, and the MGAT3 gene is a pseudogene in mice $[8,11]$. 
Recently, it was reported that mice lacking Mgat2 are protected from metabolic disorders induced by high-fat feeding and show increased energy expenditure, suggesting that MGAT2 may be a key determinant of fat absorption and energy metabolism [12]. However, the exact mechanisms of increased oxygen consumption in Mgat2-deficient mice are still unknown [12].

In this study, we constructed and analyzed Mgat2deficient mice to examine the physiological importance of MGAT2 in detail, with a focus on lipid metabolism in the small intestine. We included the heterozygote $\left(\right.$ Mgat $\left.^{+/-}\right)$mice because there were limited data on the phenotype of $\mathrm{Mgat}^{+/-}$mice [12].

\section{Results}

\section{Generation of Mgat2-deficient mice}

We generated Mgat2-deficient mice as described in Methods (Figure 1A). Mgat $2^{-/-}$mice were viable and fertile without apparent abnormalities. Mgat2 deficiency was confirmed by Southern blotting of genomic DNA (Figure 1B). Gene expression levels of Mgat2 were reduced in the duodenum of $\mathrm{Mgat}^{+/-}$mice and undetectable in $\mathrm{Mgat}^{-/-}$mice (Figure 1C). MGAT activity in the total small intestine was reduced in the Mgat2deficient mice (Figure 1D).

\section{Reduced fat absorption in the Mgat2-deficient mice}

In oral fat tolerance test (OFTT), plasma triglyceride levels were transiently increased in $\mathrm{Mgat}^{+/+}$but not in $\mathrm{Mgat}^{-/-}$mice (Figure 2), indicating inhibition of triglyceride absorption into $M g a t 2^{-/-}$mice circulation.

\section{Reduced body weight gain and adiposity in the Mgat2-deficient mice}

In order to examine the physiological significance of the inhibition of fat absorption by Mgat 2 deficiency on long-term body weight homeostasis, we challenged the Mgat2-deficient mice with an ND or HFD. On an ND, $\mathrm{Mgat}^{+/+}, \mathrm{Mgat}^{+/-}$, and $\mathrm{Mgat} 2^{-/-}$mice showed similar body weight gains (Figure 3A). In contrast, when maintained on an HFD, Mgat $2^{-/-}$mice exhibited a markedly reduced rate of body weight gain (Figure 3A). Mgat $^{-/-}$ mice showed approximately $26 \%$ lower body weight compared with $\mathrm{Mgat}^{+/+}$mice after 10 weeks of HFD feeding. $M g a t 2^{+/-}$mice had an intermediate phenotype. Analysis of body composition showed that fat mass was reduced by approximately $15 \%$ in $\mathrm{Mgat}^{+/-}$and $57 \%$ in $\mathrm{Mgat}^{-/-}$mice (Figure 3B), with no difference in lean body mass when maintained on an HFD (Figure 3C). There was no difference in total food intake based on body weight (Figure 3D). Therefore, the Mgat2-deficient mice were protected from HFD-induced obesity independent of food intake.
Improved insulin sensitivity in the Mgat2-deficient mice

No difference was observed among genotypes in plasma parameters, including blood glucose, insulin, total cholesterol, NEFA, and triglycerides in the fed state (Table 1), and in glucose tolerance (Figure 4A, B) on an ND. When maintained on an HFD, Mgat2 ${ }^{+/+}$mice showed an expected increase in blood glucose, insulin, and total cholesterol levels (Table 1) as well as in glucose intolerance (Figure 4A, B). However, insulin and total cholesterol levels were significantly reduced in $\mathrm{Mgat}^{+/-}$and $\mathrm{Mgat}^{-/-}$mice (Table 1). Furthermore, Mgat $2^{-/-}$mice exhibited improvement of glucose tolerance (Figure 4A, B) and increased insulin sensitivity as assessed by homeostasis model assessment for insulin resistance (HOMA-IR) (Figure 4C). These data suggested that the Mgat2-deficient mice were protected from HFD-induced insulin resistance and hypercholesterolemia. Subsequently, we mainly focused on the HFD-fed groups because no change was observed in the plasma parameters and body weights of the ND-fed groups.

\section{Higher oxygen consumption in the Mgat2-deficient mice}

Reduced body weight gain without alteration in food intake in the Mgat2-deficient mice suggested alterations in energy metabolism. Oxygen consumption during the light-dark phase was significantly higher in $\mathrm{Mgat}^{-/-}$ mice on an HFD (Figure 5).

\section{Lipid levels in feces and the small intestine}

We measured lipid levels in feces and the small intestine to investigate the metabolic fate of unabsorbed triglycerides as a result of Mgat2 deficiency. NEFA levels in feces was similar among genotypes (Figure 6A), and triglyceride levels in the feces were slightly increased (Figure 6B). However, fecal outputs of triglycerides were less than $1 \%$ of those contained in an HFD, indicating that the majority of ingested fat exhibited normal uptake by the enterocytes in the Mgat2-deficient mice. Triglyceride levels in the duodenum, jejunum, and ileum were not different among genotypes (Figure 6C-E). Histological examination also showed no difference in the amount of lipid droplets in the duodenum (data not shown). These results suggested that in the Mgat2-deficient mice, fat uptake into the enterocytes was normal but lipids did not accumulate in the cells.

\section{Expression levels of genes involved in fatty acid oxidation} in the small intestine and the liver

Considering the data obtained from our study, including that for reduced fat absorption into the circulation, normal fat uptake in enterocytes, and higher oxygen consumption in the Mgat2-deficient mice, we hypothesized that unabsorbed lipids were metabolized in the enterocytes of the Mgat2-deficient mice. Expression levels of 
A

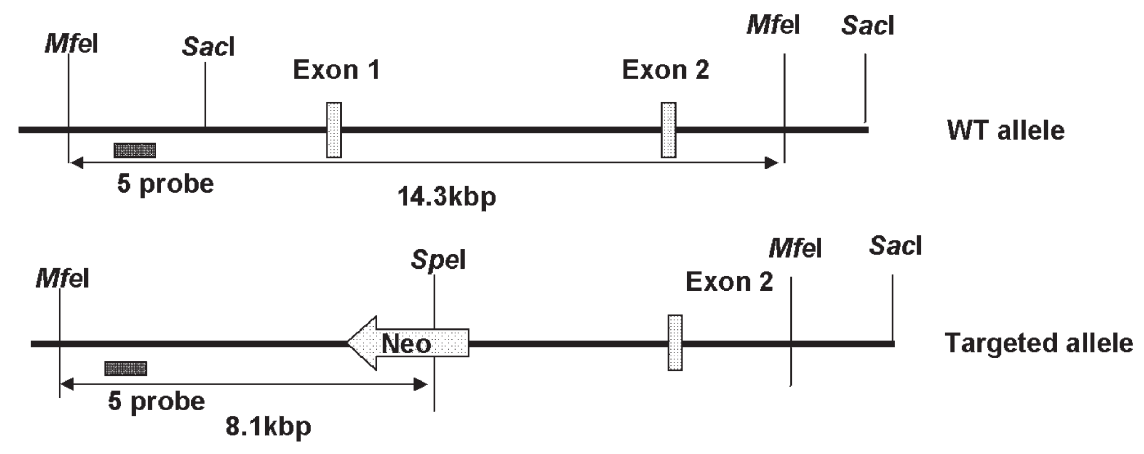

B $+/++/-\quad-/-$
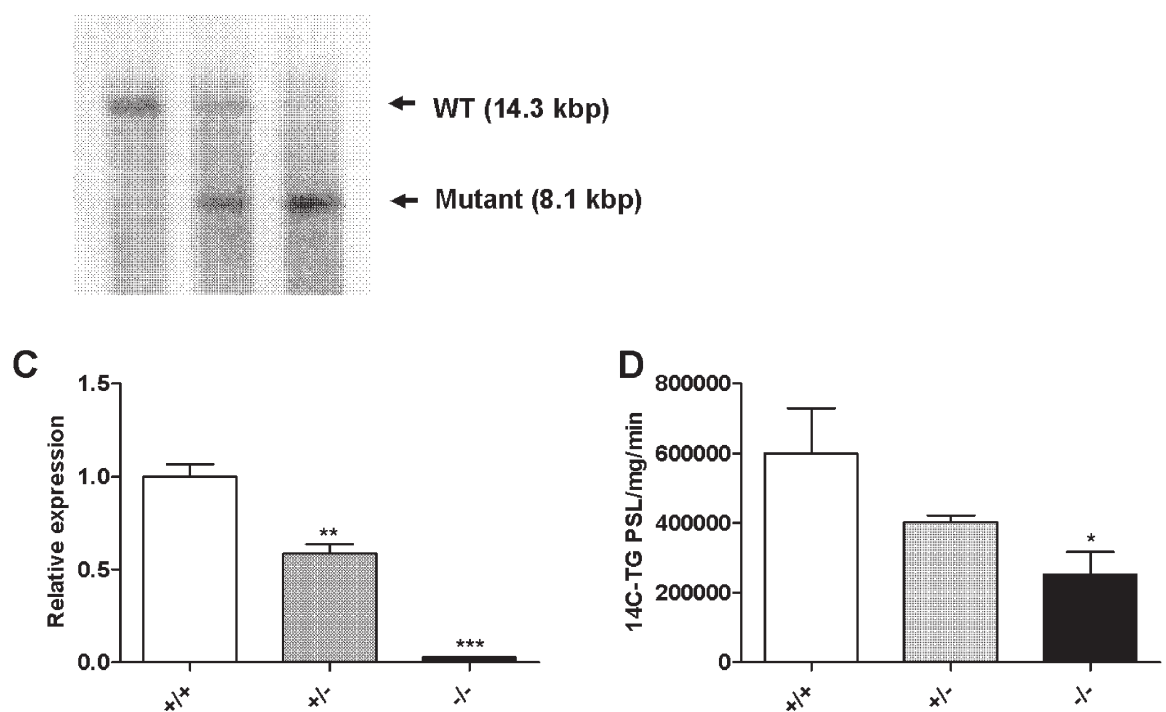

Figure 1 Generation of Mgat2-deficient mice. (A) Schematic diagram of the derivation of Mgat2-deficient mice by homologous recombination. (B) Southern blotting of genomic DNA. (C) mRNA levels of Mgat2 in the duodenum. (D) Intestinal MGAT activity. Each bar represents mean \pm SEM. $n=3-5$ per group. ${ }^{*} P<0.05,{ }^{* *} P<0.01,{ }^{* * *} P<0.001$ vs. $+/+$.

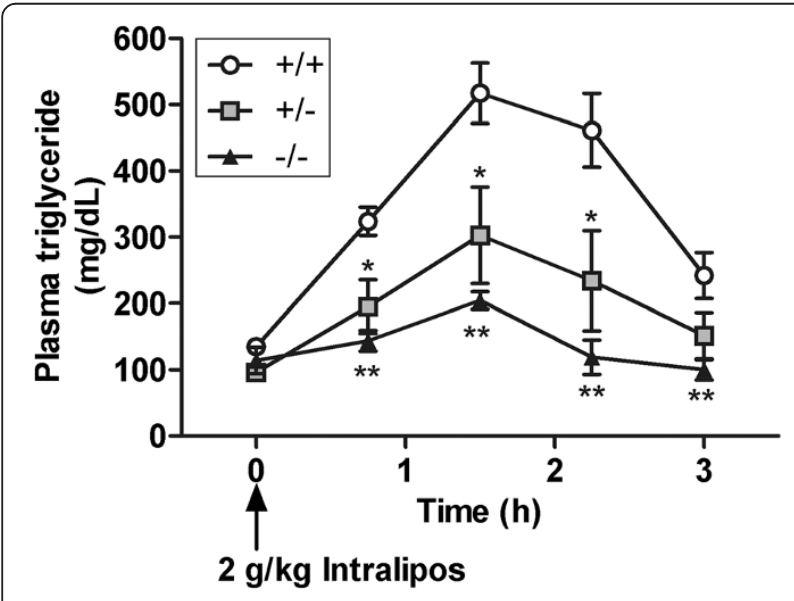

Figure 2 Reduced fat absorption in the Mgat2 deficient mice. Time course of changes in plasma triglyceride levels after an oral challenge of Intralipos. Each bar represents mean \pm SEM. $n=5-6$ per group. ${ }^{*} P<0.05,{ }^{* *} P<0.01$ vs. $+/+$ genes involved in fatty acid oxidation, such as Cpt-1a, Acox1, Hmgcs2, Acot1, Acot2, Mcad, and Lcad, were significantly upregulated in the duodenum of the Mgat2deficient mice (Figure 7). The increase of expression levels of these genes in the liver was not as obvious as that in the duodenum (Figure 8).

\section{Discussion}

In this study, we constructed novel Mgat2-deficient mice and showed that these mice were protected from HFDinduced obesity and insulin resistance in accordance with a recent report by Yen et al. [12]. OFTT showed that fat absorption into the circulation was significantly reduced in the Mgat2-deficient mice as a result of inhibition of triglyceride resynthesis in the enterocytes. Decreased fat absorption may result in protection from obesity and insulin resistance in mice fed HFD for longterm. Improvement of insulin sensitivity might be 

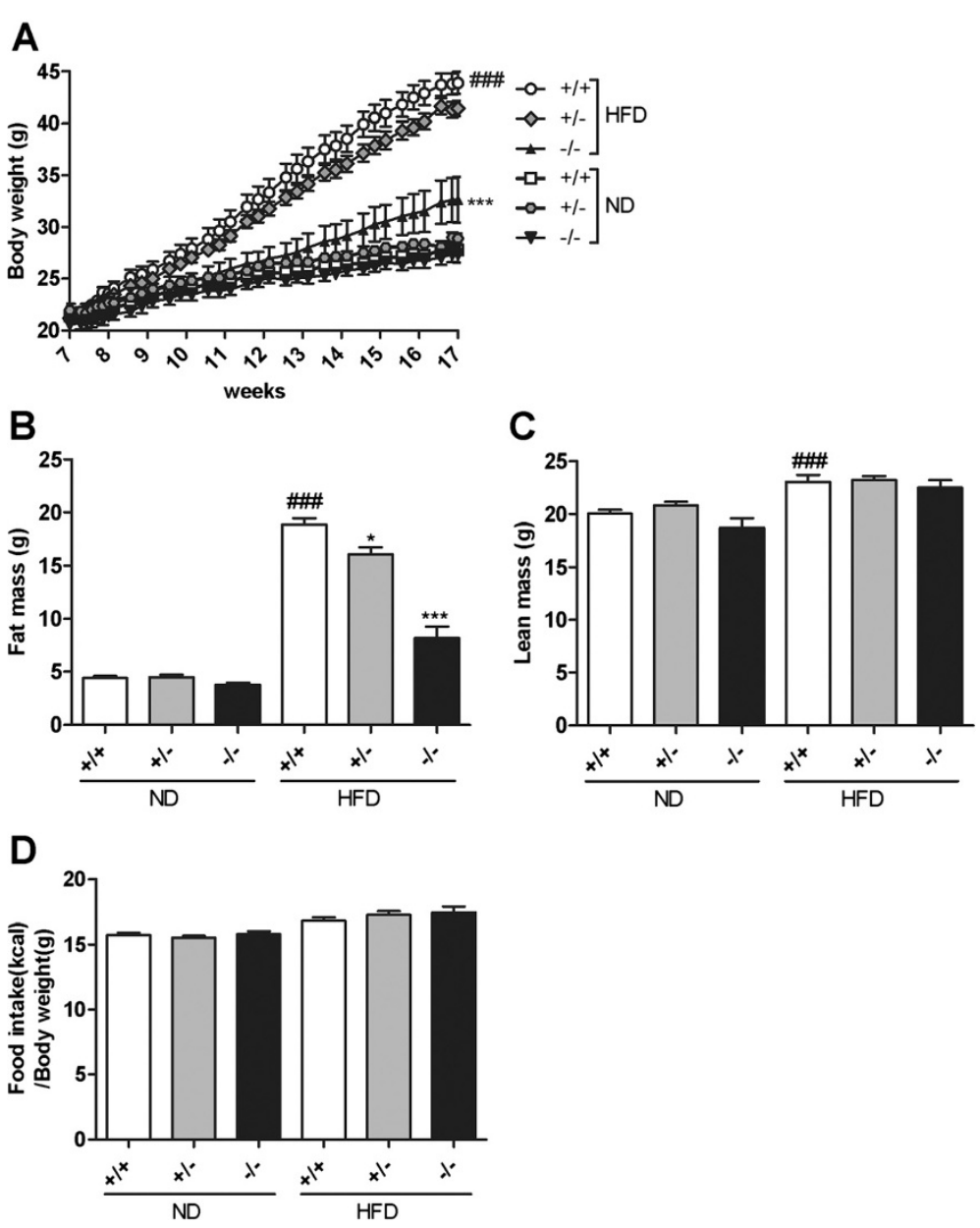

Figure 3 Reduced body weight gain and adiposity in the Mgat2-deficient mice on an HFD. Time course of changes in body weights (A), difference in fat mass (B), lean mass (C), and total food intake (D) after 10 weeks of ND or HFD feeding. Each bar represents mean \pm SEM. $\mathrm{n}=6-15$ per group. ${ }^{*} \mathrm{P}<0.05,{ }^{* * *} P<0.001$ vs. HFD $+/+.{ }^{\# \# \#} P<0.001$ vs. ND $+/+$.

explained not only by reduced weight gain but also by protection against lipotoxicity. Heterozygote $\left(\right.$ Mgat2 $\left.^{+/-}\right)$ mice had an intermediate phenotype between $\mathrm{Mgat}^{+/+}$ and $\mathrm{Mgat2}^{-/-}$and were partially protected from metabolic disorders, indicating a gene dosage-dependent effect of Mgat2 and feasibility of MGAT2 as a potential drug target.

Regarding anti-obesity drugs, orlistat is a gastric and pancreatic lipase inhibitor that reduces dietary fat absorption [13]. However, orlistat has mechanism-based gastrointestinal adverse effects, including fatty and oily stool, fecal urgency, and fecal incontinence [13]. Fatty and oily stool was not observed in the Mgat2-deficient mice in our study. Fecal levels of triglycerides and NEFAs were comparable among genotypes. Taken together, the inhibition of MGAT2 may be a safer strategy than the inhibition of pancreatic lipase for the treatment of obesity.

Table 1 Plasma parameters in the fed state of the Mgat2-deficient mice

\begin{tabular}{|c|c|c|c|c|c|c|}
\hline & ND & & & HFD & & \\
\hline Parameter & Mgat2 $^{+/+}$ & Mgat2 $^{+/-}$ & Mgat2 $^{-1-}$ & Mgat $^{+/+}$ & Mgat2 $^{+/-}$ & Mgat2 $^{-/-}$ \\
\hline Glucose (mg/dL) & $132 \pm 4$ & $130 \pm 3$ & $135 \pm 5$ & $168 \pm 6^{\# \# \#}$ & $168 \pm 5$ & $151 \pm 7$ \\
\hline Insulin (ng/mL) & $1.4 \pm 0.2$ & $1.7 \pm 0.4$ & $2.0 \pm 1.0$ & $35.8 \pm 10.4^{\# \#}$ & $14.6 \pm 3.0^{*}$ & $5.7 \pm 1.8^{*}$ \\
\hline Triglyceride (mg/dL) & $114.5 \pm 12.5$ & $123.2 \pm 12.7$ & $90.5 \pm 5.6$ & $102.7 \pm 6.5$ & $119.3 \pm 7.4$ & $94.4 \pm 7.9$ \\
\hline Total cholesterol (mg/dL) & $81 \pm 5$ & $89 \pm 4$ & $94 \pm 7$ & $196 \pm 14^{\# \# \#}$ & $137 \pm 6^{* * *}$ & $118 \pm 6^{* * *}$ \\
\hline NEFA (mEq/L) & $0.45 \pm 0.03$ & $0.43 \pm 0.04$ & $0.36 \pm 0.04$ & $0.46 \pm 0.02$ & $0.45 \pm 0.02$ & $0.46 \pm 0.03$ \\
\hline
\end{tabular}

Data are expressed as mean \pm SEM. $n=6-15$ per group. ${ }^{*} P<0.05,{ }^{* * *} P<0.001$ vs. HFD $+/+.{ }^{\# \#} P<0.01,{ }^{\# \# \#} P<0.001$ vs. ND $+/+$. 


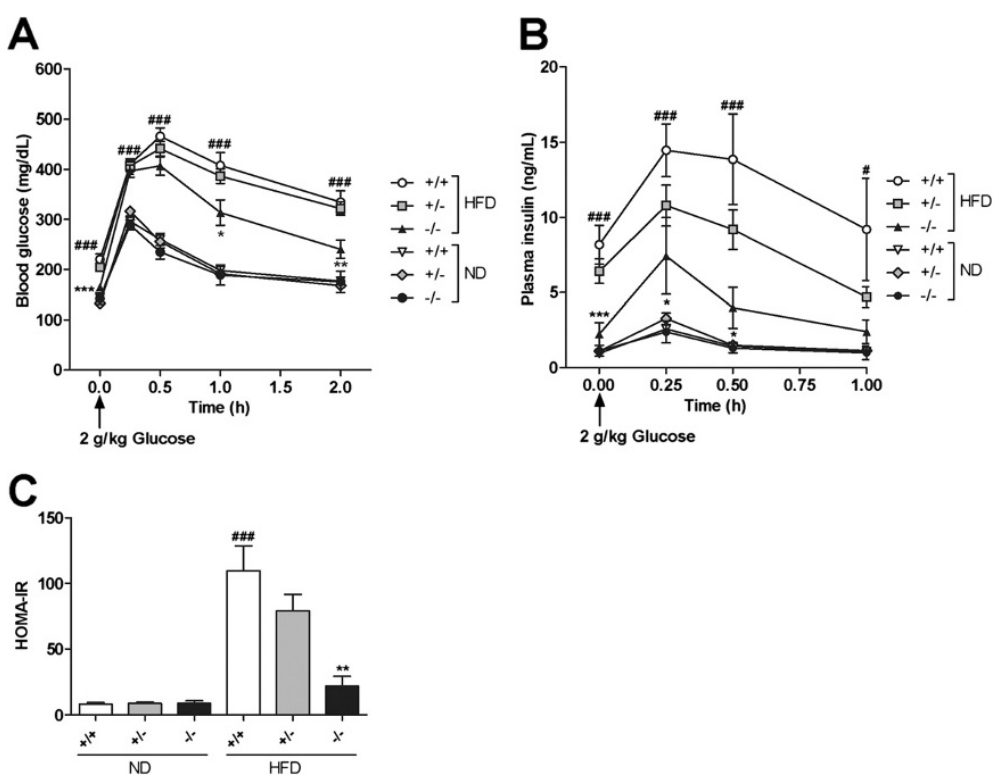

Figure 4 Improved glucose tolerance and insulin sensitivity in the Mgat2-deficient mice on an HFD. Time course of changes in blood glucose (A) and plasma insulin (B) after an oral challenge of $2 \mathrm{~g} / \mathrm{kg}$ glucose. (C) HOMA-IR was calculated. Each bar represents mean \pm SEM. $\mathrm{n}=6-15 \mathrm{per}$ group. ${ }^{*} P<0.05,{ }^{* *} P<0.01,{ }^{* * *} P<0.001$ vs. HFD $+/+.{ }^{\#} P<0.05,{ }^{\#}{ }^{\# \#} P<0.001$ vs. ND $+/+$.

Most of the triglycerides in food were absorbed into the enterocytes regardless of genotype because less than $1 \%$ of triglycerides were detected in the feces of any genotype. Reduced fat absorption into the circulation without obvious change in fat uptake into enterocytes suggested two distinct possibilities of lipid metabolism in the small intestine. One was the accumulation of lipids in the cells. However, triglyceride levels in the duodenum, jejunum, and ileum were comparable, and histological analysis also showed similar number and size of lipid droplets in the enterocytes among genotypes. Another possibility was immediate oxidation of lipids.

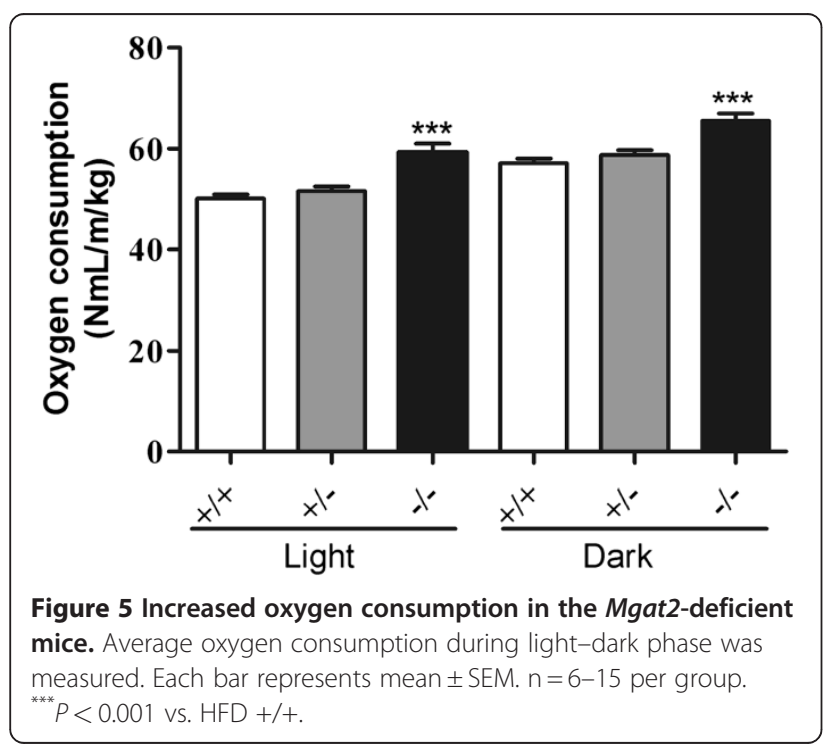

The Mgat2-deficient mice exhibited higher oxygen consumption as reported previously [12,14]. Furthermore, expression levels of genes associated with fatty acid oxidation (Cpt-1a, Acox1, Hmgcs2, Acot1, Acot2, Mcad, and $L c a d)$ were significantly upregulated in the duodenum of the Mgat2-deficient mice, whereas in the liver, increase of the gene expression levels was not as obvious as in the duodenum. Although the primary function of the small intestine is the resynthesis of triglycerides and the secretion of chylomicrons, it has been reported that the small intestine expresses oxidation-related enzymes, such as ACOX1 and MCAD, at levels comparable to those in the liver $[15,16]$ and to a significant extent contributes to the metabolic rate and daily energy expenditure [17]. It is possible that activation of fatty acid oxidation in the enterocytes may lead to higher oxygen consumption in the Mgat2-deficient mice.

Genes involved in fatty acid oxidation were reported to have peroxisome proliferator-activated receptor (PPAR) response elements in their promoter region, and NEFAs are known to be endogenous ligands of PPARa [18-22]. Therefore, upregulation of these genes may be explained by the increased levels of NEFAs, substrates of MGAT2 enzyme, and endogenous ligands of PPARa, as a result of the inhibition of triglyceride resynthesis in the enterocytes. It should be stressed that this hypothesis requires further investigation including the enzyme activity of fatty acid oxidation in the small intestine.

Yen et al. previously reported that the mechanism by which Mgat2 deficiency triggers oxygen consumption was unclear and mRNA expression of genes involved in 

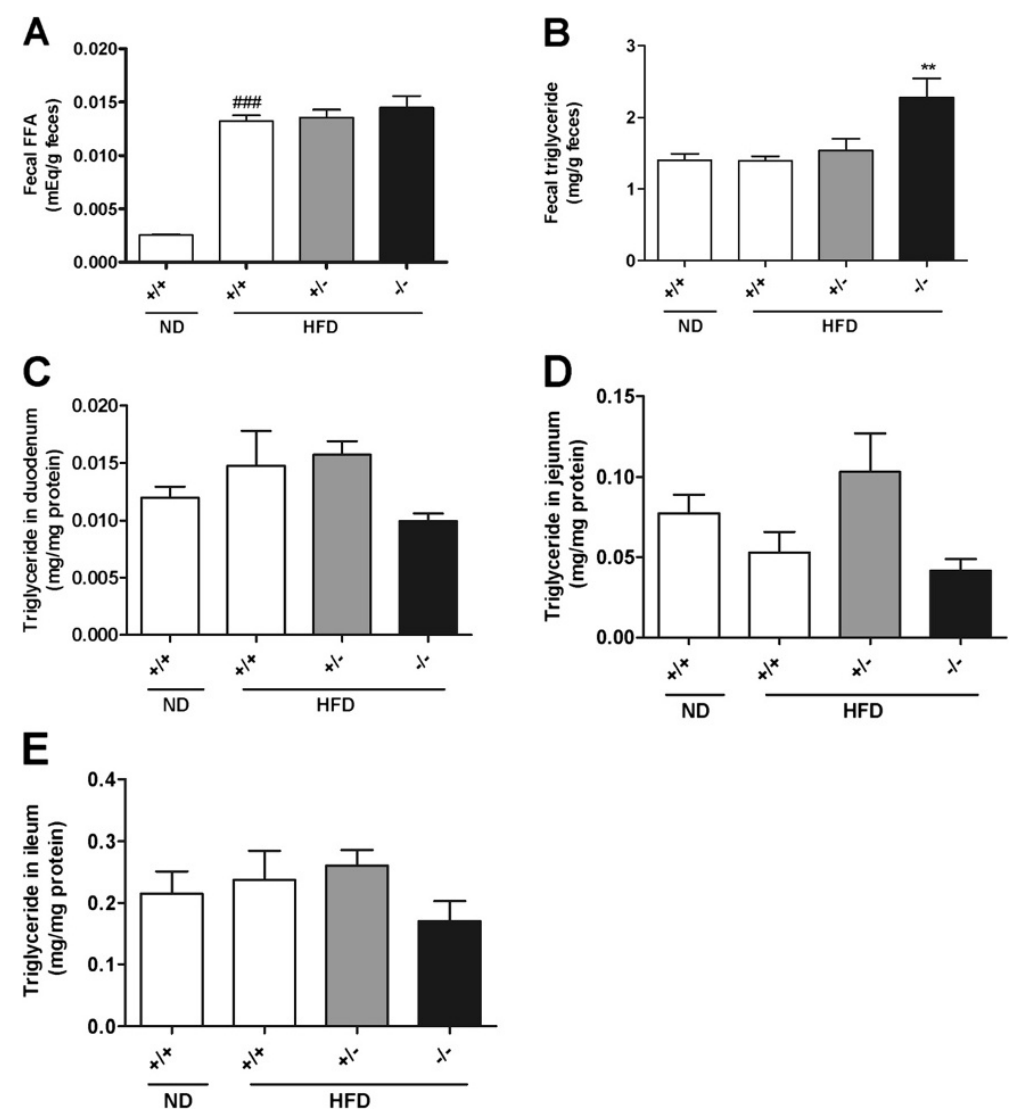

Figure 6 Lipid levels in the feces and small intestine. Fecal levels of NEFA (A) and triglycerides (B), and triglyceride levels in the duodenum (C), ileum (D), and jejunum (E) were measured. Each bar represents mean \pm SEM. $n=3-15$ per group. ${ }^{* *} P<0.01$, vs. HFD +/+. ${ }^{\# \#} P<0.001$ vs. ND +/+.

fat oxidation in the small intestine and brown adipose tissue showed no differences among genotypes [12]. The difference in gene expression profiles in the small intestine between Yen's study and our results could be explained by the measurement position used. We analyzed mRNA expression in the duodenum, whereas the jejunum was used in the study by Yen et al.

In mice, MGAT2 is mainly expressed in the small intestine. In contrast, in humans, MGAT2 is highly expressed both in the small intestine and liver[8,23]. It is well known that the hepatocytes play a key role in lipid metabolism by accumulating triglycerides and oxidizing fatty acid. Excessive storage and accumulation of lipid droplets into the hepatocytes result in hepatic steatosis. Therefore, the inhibition of MGAT2 activity might lead to lipid oxidation in the hepatocytes and improvement of fatty liver in humans.

\section{Conclusions}

In summary, the Mgat2-deficient mice are protected from HFD-induced obesity and insulin resistance. MGAT2 has a pivotal role in lipid metabolism in the small intestine, and the inhibition of MGAT2 activity may be a promising strategy for the treatment of obesity and type 2 diabetes.

\section{Methods}

Generation of Mgat2-deficient mice

Mgat2-targeted 129/SvJae embryonic stem cells and, subsequently, mice carrying this mutation with a targeting vector designed to replace exon 1 of Mgat2 with a neomycin-resistance cassette (Figure 1A) were generated. Mgat 2 deficiency was confirmed by Southern blotting of genomic DNA, which was digested with MfeI and SoeI using a probe hybridizing to sequences upstream of those included in the targeting vector, and by quantitative PCR. Mgat2 $2^{-1-}$ and wild-type littermates were generated by breeding heterozygotes that were first backcrossed with C57BL/6 J mice. Mice were housed in a pathogen-free facility. Diets included a normal diet (ND, CRF-1, Oriental Yeast Co., $3.59 \mathrm{kcal} \mathrm{g}^{-1}$ ) and a high-fat diet (HFD, Oriental Yeast Co., 59\% cal fat (lard), $5.578 \mathrm{kcal} / \mathrm{g}$ ). Animals were maintained in a 12/12-h light-dark cycle. All experiments were performed on 


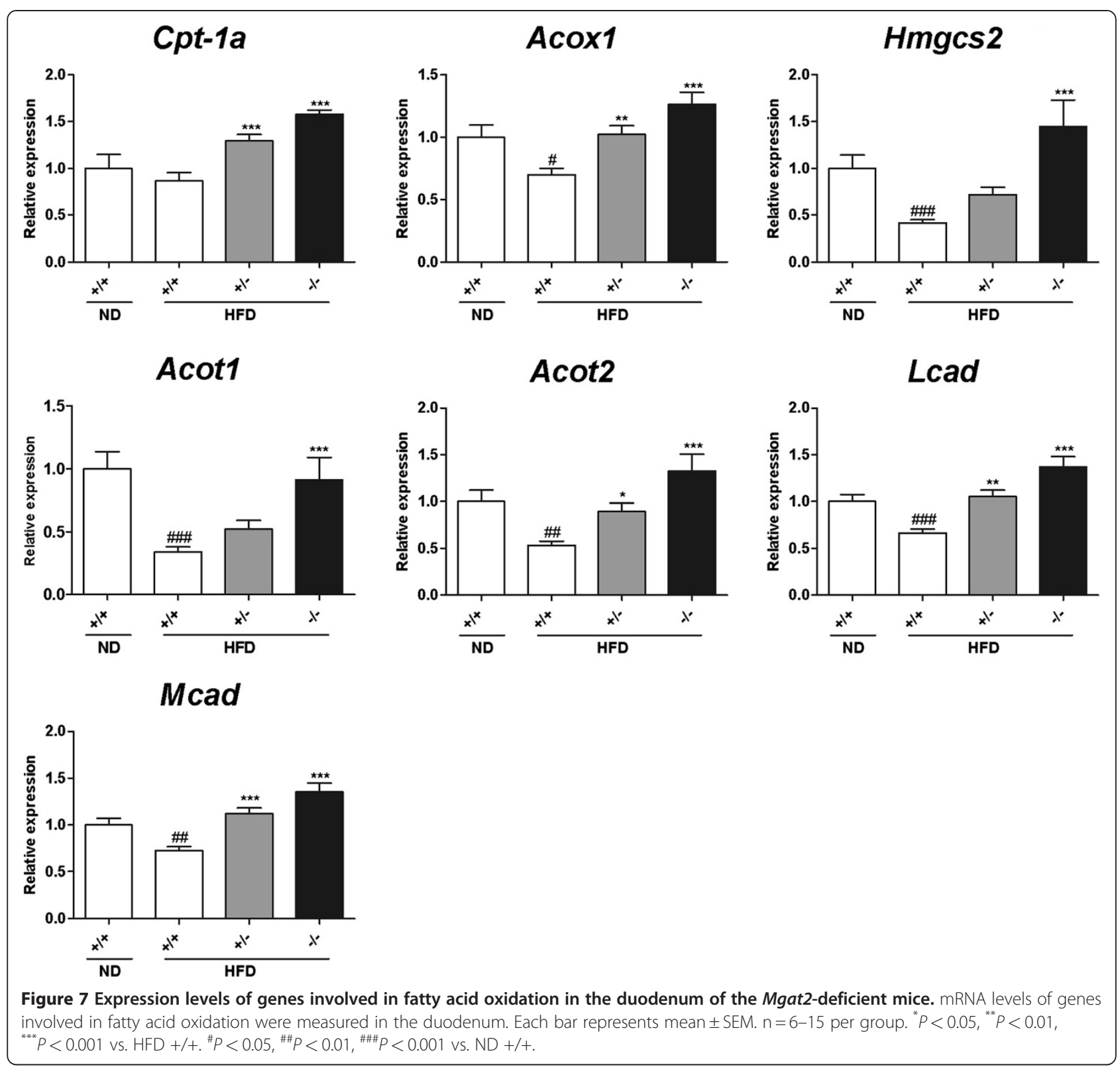

male animals. Experimental protocols concerning the use of laboratory animals were reviewed and endorsed by the Institutional Animal Care and Use Committee of Mitsubishi Tanabe Pharma Corporation.

\section{MGAT enzyme assays}

MGAT activity was determined by measuring the incorporation of the $\left[{ }^{14} \mathrm{C}\right]$ palmitoyl moiety into triacylglycerol with $\left[{ }^{14} \mathrm{C}\right]$ palmitoyl-CoA (ARC, St. Louis, MO) and 2-monooleoylglycerol. Crude membranes $(10 \mu \mathrm{g})$ of the small intestine were used as the enzyme source. Assays were performed in $200 \mu \mathrm{l}$ buffer $(100 \mathrm{mM}$ Tris$\mathrm{HCl}, \mathrm{pH} 7.4,200 \mathrm{mM}$ sucrose, $5 \mathrm{mM} \mathrm{MgCl}, 1.25 \mathrm{mg} / \mathrm{ml}$ BSA) containing $10 \mu \mathrm{M} 2$-monooleoylglycerol and $20 \mu \mathrm{M}$ $\left[{ }^{14} \mathrm{C}\right]$ palmitoyl-CoA. Reactions were performed for $5 \mathrm{~min}$ at $30^{\circ} \mathrm{C}$ and the products were extracted with $1 \mathrm{ml}$ of chloroform:methanol $(2: 1, \mathrm{v} / \mathrm{v})$. The extracts were dried and separated by thin layer chromatography with hexane: diethyl ether:acetic acid (80:20:1, v/v/v). Areas containing $\left[{ }^{14} \mathrm{C}\right]$ triacylglycerol were visualized and quantitated using the FLA3000 fluorescence detection system (Fujifilm, Tokyo, Japan).

\section{Oral fat tolerance test (OFTT)}

Mice were fasted overnight and then $10 \mathrm{ml} / \mathrm{kg}$ of Intralipos containing $20 \%$ soybean oil (v/v) (Otsuka, Tokyo, Japan) was orally administered. Plasma samples were obtained from the tail vein before and 75, 150, 225, and 


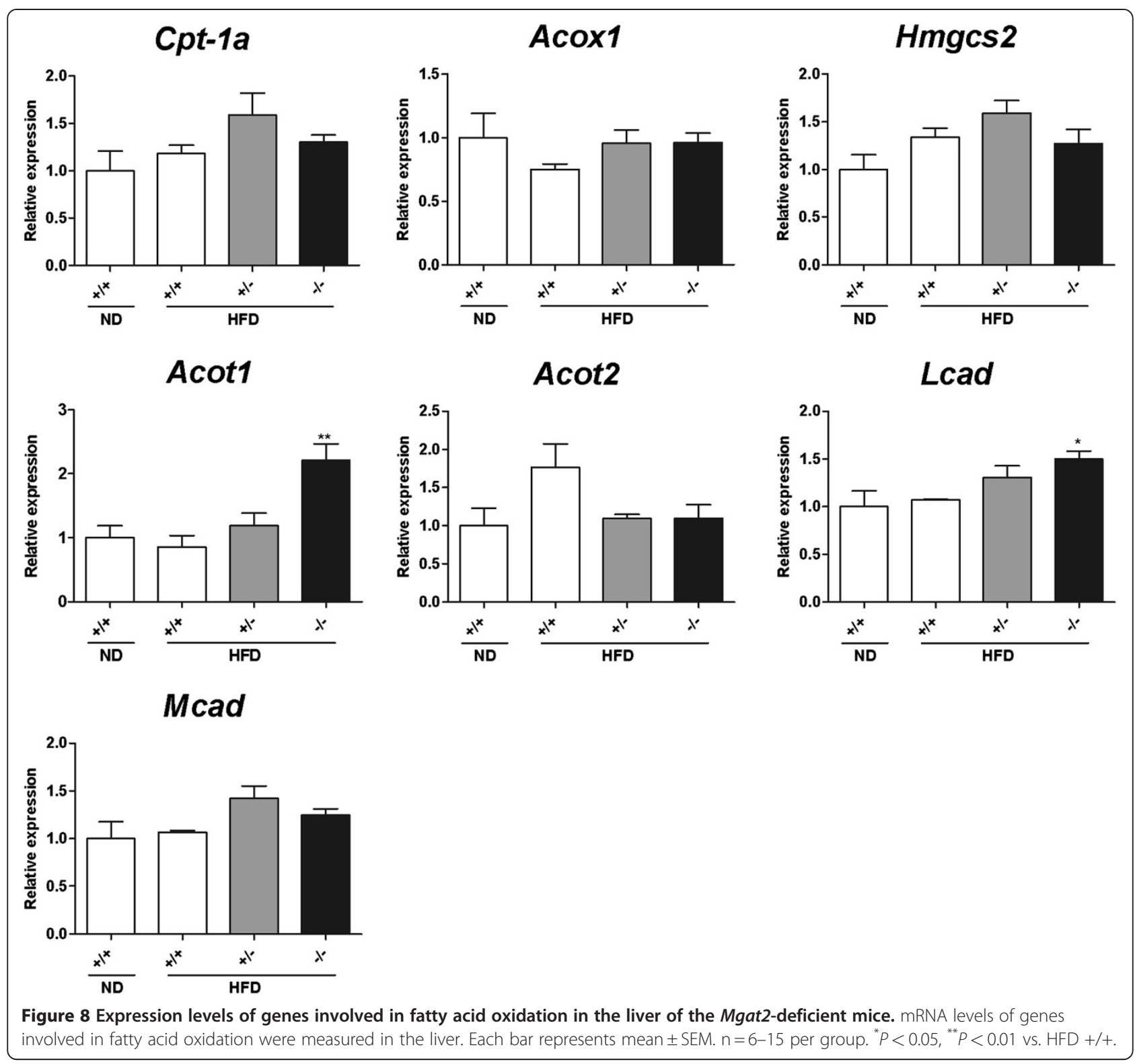

$300 \mathrm{~min}$ after the fat challenge for determination of plasma triglyceride levels.

\section{Long-term feeding studies}

Animals were weaned at three weeks of age and maintained on an ND or switched to an HFD at seven weeks of age. They were weighed and their food intake was monitored. After 10 weeks of HFD feeding, blood samples in the fed state and feces were collected for 3 days. After overnight fasting, the mice were killed by whole blood collection from the abdominal aorta under ether anesthesia. The small intestine and liver were quickly removed from each mouse, immediately frozen in liquid nitrogen, and stored at $-80^{\circ} \mathrm{C}$ for quantitative PCR and lipid measurements.

\section{Determination of body composition}

After 10-12 weeks of HFD feeding, the body composition was analyzed by dual-energy X-ray absorptiometry with PIXImus2 (GE Healthcare, Tokyo, Japan) under ether anesthesia.

\section{Determination of plasma parameters}

Plasma triglyceride, total cholesterol, and non-esterified fatty acid (NEFA) levels were determined using an enzymatic assay kit (Wako Chemicals, Osaka, Japan). Blood glucose was determined using commercially available kits based on the glucose oxidase method (Wako Chemicals, Osaka, Japan). Plasma insulin was assayed using an enzyme-linked immunosorbent assay (ELISA) kit (Morinaga Co. Ltd., Yokohama, Japan). 


\section{Oral glucose tolerance test (OGTT)}

An oral glucose tolerance test (OGTT) was performed after 10 weeks of HFD feeding. Mice were fasted for $3 \mathrm{~h}$ and then $2 \mathrm{~g} / \mathrm{kg}$ glucose solution was orally administered at a volume of $10 \mathrm{ml} / \mathrm{kg}$. Blood samples were obtained from the tail vein before and 15, 30, 60, and $120 \mathrm{~min}$ after the glucose challenge for determination of blood glucose levels. Plasma samples were obtained before and 15,30 , and $60 \mathrm{~min}$ after the glucose challenge for determination of plasma insulin levels. HOMA-IR was calculated [24].

\section{Determination of lipid levels in feces and the small intestine}

Fecal lipids were extracted with a 30 -fold volume of chloroform:methanol $(2: 1, \mathrm{v} / \mathrm{v})$ with vigorous shaking. Then, a 1/3.75-fold volume of water was added and the mixture was shaken vigorously. The organic phase was collected and again a 30-fold volume of chloroform: methanol $(2: 1, \mathrm{v} / \mathrm{v})$ was added to the water phase. After vigorous shaking, the organic phase was collected, and the combined organic phase was evaporated. The dried residue was dissolved in isopropanol:Triton X-100 (9:1, $\mathrm{v} / \mathrm{v})$ and measured for triglyceride and NEFA levels using an enzymatic assay kit. The small intestine was homogenized in 20 -fold volume of saline. Then, lipids were extracted with a 3.75-fold volume of chloroform: methanol $(2: 1, \mathrm{v} / \mathrm{v})$ with vigorous shaking. The organic phase was collected and evaporated. The dried residue was dissolved in isopropanol:Triton X-100 $(9: 1, \mathrm{v} / \mathrm{v})$ and measured for triglyceride levels using an enzymatic assay kit.

\section{Determination of oxygen consumption}

After 10 weeks of HFD feeding, oxygen consumed by each mouse was measured using an ARCO-2000 analyzer (Arco System, Chiba, Japan) every 3 min for a 24-h period.

\section{Histology}

Portions of duodenum were fixed in $10 \%$ (v/v) neutral buffered formalin and embedded in paraffin. Paraffin sections (3-5 $\mu \mathrm{m}$ thick) were stained with hematoxylin and eosin and examined under a light microscope.

\section{Quantitative PCR}

Total RNA of the duodenum and the liver was extracted without using DNase. Total RNA was reverse transcribed by TaqMan Reverse Transcriptase Reagents (Applied Biosystems, Foster City, CA). Real-time PCR was performed with SYBR Green PCR mix (Applied Biosystems, Foster City, CA) and analyzed with an ABI Prism 7000 Sequence Detection System (Applied Biosystems, Foster City, CA). The relative expression levels were compared after normalization to B-actin. The primers used for B-actin and Mgat2 were from QIAGEN (Tokyo, Japan). The following primer pairs were used ( $5^{\prime}$ to $\left.3^{\prime}\right)$ : Cpt-1a, forward: AAGCTGTTCAAGATAGCTTG, reverse: TGCTGATGACGGCTATGGTGT; Acot1, forward: GGCTGGGAATGGAGTTTCAT, reverse: GCTATCCAA GAAAAGTGCCAGG; Acot2, forward: AGTGCCTATGA AGGACTGAGGA, reverse: GGTAAAGGTGCTTTCTG CC; Acox1, forward: GGTAAAGGTGCTTTCTGCC, reverse: AGATAAACTCCCCAAGATTCAAGAC; Hmgcs2, forward: TGTCCCCTGAGGAATTCACAGAA, reverse: AACGAGTGGATGAGATGCATCG; $L c a d$, forward: CTG GTTAAGTGATCTCGTGATCGTCG, reverse: CTGGCA ATCGGACATCTTCAAAGAATAGT; $M c a d$, forward GC TCTGATGTGGCGGCCATTA, reverse: AAGGCTTTAC TAGCGGGTACTTTAGG

\section{Statistical analysis}

All data were expressed as mean \pm SEM. The comparison of mean values between two groups was performed by the Student's t-test, and the Dunnett's test was used for comparing more than two groups. $P$ values of $<0.05$ were considered statistically significant.

\section{Abbreviations}

MGAT: Acyl-coenzyme A:monoacylglycerol acyltransferase; DGAT: Acylcoenzyme A:diacylglycerol acyltransferase; ND: Normal diet; HFD: High-fat diet; OFTT: Oral fat tolerance test; OGTT: Oral glucose tolerance test; HOMA-IR: Homeostasis model assessment for insulin resistance;

NEFA: Non-esterified fatty acid; ELISA: Enzyme-linked immunosorbent assay; Cpt-1a: Carnitine palmitoyltransferase-1a; Acox1: Acyl-coenzyme A oxidase 1; Hmgcs2: 3-hydroxy-3-methyl-glutaryl-coenzyme A synthase 2; Acot: Acylcoenzyme A thioesterase; Mcad: Medium-chain acyl dehydrogenase;

Lcad: Long-chain acyl dehydrogenase; PPAR: Peroxisome proliferator-activated receptor.

\section{Competing interests}

The authors declare that they have no competing interests. The authors alone are responsible for the content and writing of the paper.

\section{Acknowledgments}

We thank Dr. Kenji Arakawa, Dr. Kaoru Sakai, and Dr. Masaharu Tanaka for helpful discussions through the experiments; Dr. Koji Oda, Noriko Hosoda, and Tomoko Yamashita for excellent technical assistance.

\section{Author details}

'Department I, Pharmacology Research Laboratories II, Research Division, Mitsubishi Tanabe Pharma Corporation, 2-2-50, Kawagishi, Toda-shi, Saitama 335-8505, Japan. ${ }^{2}$ Discovery molecular pharmacology Department, Discovery Screening Center, Research Division, Mitsubishi Tanabe Pharma Corporation, Saitama, Japan. ${ }^{3}$ Safety Department II, Safety Research Laboratory, Research Division, Mitsubishi Tanabe Pharma Corporation, Chiba, Japan. ${ }^{4}$ Target Discovery and Biomarker Research Department, Advanced Medical Research Laboratories, Research Division, Mitsubishi Tanabe Pharma Corporation, Yokohama, Japan.

\section{Authors' contributions}

$\Pi$ designed the study, carried out the animal experiments and quantitative PCR, and drafted the manuscript. SF, NT, TI carried out the animal experiments. HA and KW carried out the generation of Mgat2-deficient mice. HS carried out the histological analysis. NO and MS participated in the design of the study. AO conceived of the study, and participated in its design and coordination and helped to draft the manuscript. All authors read and approved the final manuscript. 
Received: 13 March 2012 Accepted: 27 April 2012

Published: 14 June 2012

\section{References}

1. Ros E: Intestinal absorption of triglyceride and cholesterol. Dietary and pharmacological inhibition to reduce cardiovascular risk. Atherosclerosis 2000 151:357-379.

2. Lehner R, Kuksis A: Biosynthesis of triacylglycerols. Progress in lipid research 1996, 35:169-201.

3. Kayden HJ, Senior JR, Mattson FH: The monoglyceride pathway of fat absorption in man. The Journal of clinical investigation 1967, 46:1695-1703.

4. Phan CT, Tso P: Intestinal lipid absorption and transport. Frontiers in bioscience: a journal and virtual library 2001, 6:D299-319.

5. Coleman RA, Haynes EB: Monoacylglycerol acyltransferase. Evidence that the activities from rat intestine and suckling liver are tissue-specific isoenzymes. The Journal of biological chemistry 1986, 261:224-228.

6. Cheng D, Nelson TC, Chen J, Walker SG, Wardwell-Swanson J, Meegalla R, Taub R, Billheimer JT, Ramaker M, Feder JN: Identification of acyl coenzyme A:monoacylglycerol acyltransferase 3, an intestinal specific enzyme implicated in dietary fat absorption. The Journal of biological chemistry 2003, 278:13611-13614.

7. Cao J, Lockwood J, Burn P, Shi Y: Cloning and functional characterization of a mouse intestinal acyl-CoA:monoacylglycerol acyltransferase, MGAT2. The Journal of biological chemistry 2003, 278:13860-13866

8. Yen CL, Farese RV Jr: MGAT2, a monoacylglycerol acyltransferase expressed in the small intestine. The Journal of biological chemistry 2003, 278:18532-18537.

9. Yen CL, Stone SJ, Cases S, Zhou P, Farese RV Jr: Identification of a gene encoding MGAT1, a monoacylglycerol acyltransferase. Proceedings of the National Academy of Sciences of the United States of America 2002, 99:8512-8517.

10. Cao J, Burn P, Shi Y: Properties of the mouse intestinal acyl-CoA: monoacylglycerol acyltransferase, MGAT2. The Journal of biological chemistry 2003, 278:25657-25663.

11. Yue YG, Chen YQ, Zhang Y, Wang H, Qian YW, Arnold JS, Calley JN, Li SD, Perry WL, 3rd, Zhang HY, et al: The Acyl CoenzymeA:Monoacylglycerol Acyltransferase 3 (MGAT3) Gene is a Pseudogene in Mice but Encodes a Functional Enzyme in Rats. Lipids 2011

12. Yen CL, Cheong ML, Grueter C, Zhou P, Moriwaki J, Wong JS, Hubbard B, Marmor S, Farese RV Jr: Deficiency of the intestinal enzyme acyl CoA: monoacylglycerol acyltransferase- 2 protects mice from metabolic disorders induced by high-fat feeding. Nature medicine 2009, 15:442-446

13. Padwal RS, Majumdar SR: Drug treatments for obesity: orlistat, sibutramine, and rimonabant. Lancet 2007, 369:71-77.

14. Nelson DW, Gao Y, Spencer NM, Banh T, Yen CL: Deficiency of MGAT2 increases energy expenditure without high-fat feeding and protects genetically obese mice from excessive weight gain. Journal of lipid research 2011, 52:1723-1732.

15. Kelly DP, Gordon Jl, Alpers R, Strauss AW: The tissue-specific expression and developmental regulation of two nuclear genes encoding rat mitochondrial proteins. Medium chain acyl-CoA dehydrogenase and mitochondrial malate dehydrogenase. The Journal of biological chemistry 1989, 264:18921-18925.

16. Nemali MR, Usuda N, Reddy MK, Oyasu K, Hashimoto T, Osumi T, Rao MS, Reddy JK: Comparison of constitutive and inducible levels of expression of peroxisomal beta-oxidation and catalase genes in liver and extrahepatic tissues of rat. Cancer research 1988, 48:5316-5324.

17. Krebs HA: Body size and tissue respiration. Biochimica et biophysica acta 1950, 4:249-269.

18. Mallordy A, Poirier H, Besnard P, Niot I, Carlier H: Evidence for transcriptional induction of the liver fatty-acid-binding-protein gene by bezafibrate in the small intestine. European journal of biochemistry / FEBS 1995, 227:801-807

19. Mochizuki K, Suruga K, Yagi E, Takase S, Goda T: The expression of PPARassociated genes is modulated through postnatal development of PPAR subtypes in the small intestine. Biochimica et biophysica acta 2001, 1531:68-76.

20. Barclay TB, Peters JM, Sewer MB, Ferrari L, Gonzalez FJ, Morgan ET: Modulation of cytochrome P-450 gene expression in endotoxemic mice is tissue specific and peroxisome proliferator-activated receptor-alpha dependent. The Journal of pharmacology and experimental therapeutics 1999, 290:1250-1257.
21. Chatelain F, Kohl C, Esser V, MCGarry JD, Girard J, Pegorier JP: Cyclic AMP and fatty acids increase carnitine palmitoyltransferase I gene transcription in cultured fetal rat hepatocytes. European journal of biochemistry / FEBS 1996, 235:789-798.

22. Rodriguez JC, Gil-Gomez G, Hegardt FG, Haro D: Peroxisome proliferatoractivated receptor mediates induction of the mitochondrial 3-hydroxy-3 -methylglutaryl-CoA synthase gene by fatty acids. The Journal of biological chemistry 1994, 269:18767-18772.

23. Lockwood JF, Cao J, Burn P, Shi Y: Human intestinal monoacylglycerol acyltransferase: differential features in tissue expression and activity. American journal of physiology Endocrinology and metabolism 2003, 285: E927-937.

24. Kim MK, Chae YN, Son MH, Kim SH, Kim JK, Moon HS, Park CS, Bae MH, Kim E, Han T, et al: PAR-5359, a well-balanced PPARalpha/gamma dual agonist, exhibits equivalent antidiabetic and hypolipidemic activities in vitro and in vivo. European journal of pharmacology 2008, 595:119-125.

doi:10.1186/1476-511X-11-75

Cite this article as: Tsuchida et al: MGAT2 deficiency ameliorates highfat diet-induced obesity and insulin resistance by inhibiting intestinal fat absorption in mice. Lipids in Health and Disease 2012 11:75.

\section{Submit your next manuscript to BioMed Central and take full advantage of:}

- Convenient online submission

- Thorough peer review

- No space constraints or color figure charges

- Immediate publication on acceptance

- Inclusion in PubMed, CAS, Scopus and Google Scholar

- Research which is freely available for redistribution

Submit your manuscript at www.biomedcentral.com/submit
C) Biomed Central 